\title{
Processo de construção coletiva da Clínica Ampliada na Odontologia por meio de uma gestão colegiada
}

\author{
Najara Barbosa Rocha*; Vanessa Cristina Veltrini*; Carina Gisele Costa Bispo*; Neli Pielarisi*; Raquel Sano \\ Suga Terada*; Mitsue Fujimaki* \\ * Docente do Departamento de Odontologia da Universidade \\ Estadual de Maringá
}

Recebido em 31/08/2017. Aprovado em 14/12/2017.

\begin{abstract}
RESUMO
O objetivo deste estudo foi relatar o processo de construção coletiva da Clínica Ampliada (CA) no Departamento de Odontologia da Universidade Estadual de Maringá (UEM). Este é um estudo do tipo descritivo, de caráter documental, baseado na análise dos temas registrados nas atas das reuniões semanais da Comissão Permanente de Avaliação da Clínica Ampliada (COPACA), desde o primeiro semestre de 2010 até o primeiro semestre de 2017. Os dados foram interpretados pelo método da análise de conteúdo preconizado por Bardin. Os documentos apontaram que os impactos da gestão colegiada sobre o funcionamento da clínica odontológica da UEM foram: maior resolutividade dos problemas, menor dificuldade nos enfrentamentos dos desafios encontrados, maior comprometimento dos envolvidos, planejamento integral dos casos e satisfação dos docentes, discentes, agentes universitários e usuários. Como produtos das discussões realizadas pela COPACA, podemos citar: Prontuário único; Manual da Clínica Ampliada e Instrutivo da Clínica Ampliada. Este estudo permitiu concluir que a construção e o aprimoramento da CA no Departamento de Odontologia da Universidade Estadual de Maringá (UEM) têm ocorrido de forma contínua no período de 2010 a 2017, resultado de um esforço e sinergia da equipe. As reuniões semanais da COPACA são o espaço onde a gestão colegiada se consolida e a escuta e as potencialidades são legitimadas. Diversas temáticas emergiram a partir de problemas detectados no dia a dia. Houve discussão e reflexão e propostas foram elaboradas objetivando a melhoria do serviço e do ensino, conforme preconiza a Política Nacional de Humanização.
\end{abstract}

Descritores: Humanização da Assistência. Odontologia. Sistema Único de Saúde.

\section{INTRODUÇÃO}

De acordo com as Diretrizes Curriculares Nacionais para os cursos de Odontologia, o cirurgião-dentista deve ter um perfil generalista, humanista, crítico e reflexivo, bem como atuar em todos os níveis de atenção à saúde, com rigor técnico e científico ${ }^{1}$. Este perfil profissional humanizado é de difícil conceituação, devido ao seu caráter subjetivo, complexo e multidimensional.

Inserida no contexto da saúde, a humanização transcende a habilidade clínica dos 
profissionais, pois exige mudanças na qualidade do comportamento ${ }^{2}$. Humanizar o atendimento clínico implica em compreender as angústias e incertezas do indivíduo que o receberá, concedendo-lhe apoio e atenção permanentes na busca pelo restabelecimento e/ou manutenção da saúde. Portanto, é possível inferir que humanização é um processo que se encontra em constante transformação e que sofre influência do contexto local ${ }^{2}$ e da compreensão individual e coletiva do espaço que o outro ocupa, na relação profissional-paciente. Assim, seria o processo de transformar o relacionamento historicamente verticalizado pelo saber e poder do profissional, para um relacionamento horizontal, com trocas de conhecimento e aprendizagem mútua, escuta qualificada, visando a implementação das mudanças necessárias na forma de viver e lidar com os problemas de saúde.

A política Nacional de Humanização $(\mathrm{PNH})^{3}$, publicada em 2004, objetiva reforçar os princípios do Sistema Único de Saúde, no qual o profissional tem a responsabilidade de atuar de forma integral e resolutiva, na prevenção, reabilitação e promoção da saúde ${ }^{3}$. Essa política estimula a comunicação entre gestores, trabalhadores e usuários, de modo a construir processos coletivos de enfrentamento dos desafios no trabalho, além de basear-se no desenvolvimento da autonomia do usuário, bem como a corresponsabilidade do mesmo em relação a sua saúde ${ }^{2}$. Uma das propostas da $\mathrm{PNH}$ envolve a implantação da CA nos serviços de saúde, cujo foco principal é a atenção individual e coletiva, possibilitando que outros aspectos do sujeito, que não apenas o biológico, possam ser compreendidos e trabalhados ${ }^{4}$. Outra proposta é a questão do acolhimento, que é uma postura ética que implica na escuta do usuário em suas queixas ${ }^{5}$.

As políticas macro de indução de mudança na formação profissional na área da saúde, baseadas em movimentos internacionais e, posteriormente, nacionais, foram balizadoras para os avanços no Curso de Odontologia da Universidade Estadual de Maringá (UEM).

O projeto político-pedagógico deste curso foi discutido e reformulado em 1992, tendo como base um currículo integrado, no qual se colocou em prática, com grandes desafios, avanços e retrocessos, um processo inovador na formação de um futuro profissional da Odontologia: líder, comunicador, questionador, reflexivo, ético, capaz de atuar no sistema de saúde vigente e de pautar suas decisões clínicas na melhor evidência científica ${ }^{6}$.

O Programa de Reorientação na Formação Profissional na Saúde (Pró-Saúde), instituído por meio da Portaria Interministerial 2.101/2005, foi um grande propulsor desta proposta no Curso de Odontologia da UEM. Esta foi uma iniciativa conjunta dos Ministérios da Saúde e da Educação, apoiada pela Organização Panamericana de Saúde ${ }^{7}$. Concomitantemente, a Política Nacional de Humanização $(\mathrm{PNH})^{3}$ e a Política de Educação Permanente $(\mathrm{PEP})^{8}$ atuaram como norteadoras das discussões e da sensibilização para as mudanças, gerando a conscientização do corpo docente e técnico. A formação dos profissionais de saúde não pode apenas objetivar a busca eficiente de evidências ao diagnóstico, cuidado, tratamento, prognóstico, etiologia e profilaxia das doenças e agravos, mas também desenvolver condições de atendimento às necessidades de saúde das pessoas e das populações, da gestão setorial e do controle social em saúde, redimensionando o desenvolvimento da autonomia das pessoas até a condição de influência na formulação de políticas do cuidado. É papel das instituições de ensino, junto com o SUS, coletar, sistematizar, analisar e interpretar permanentemente informações da realidade, problematizar o trabalho e as organizações de saúde e de ensino, construindo significados e 
práticas a partir da orientação da sociedade, mediante participação ativa dos gestores, formadores, usuários e estudantes ${ }^{9}$.

No processo de construção da CA, várias atividades foram realizadas para fomentar e criar uma massa crítica motivada, desde palestras com convidados pertencentes à $\mathrm{PNH}$ e à $\mathrm{PEP}$, até a aproximação com a Coordenação Geral de Saúde Bucal, o estímulo à participação de docentes, agentes universitários, discentes e comunidade em eventos regionais e nacionais, a presença de apoiadores da PNH assessorando a condução dos trabalhos e, ainda, a organização de oficinas e grupos de trabalhos com temáticas específicas. Com apoio financeiro e diretrizes norteadoras claras, o Projeto Pró-Saúde estimulou mudanças significativas no Curso de Odontologia da UEM.

Neste contexto, em 2006, a implementação de atividades coordenadas pela área de Saúde Coletiva introduziu os alunos nas equipes de saúde bucal da Estratégia Saúde da Família (ESF) no município de Maringá/PR, a partir da territorialização, visitas domiciliares, reconhecimento dos espaços sociais, busca ativa, identificação dos riscos e vulnerabilidades, participação em reuniões do conselho local de saúde e aproximação da comunidade por meio da organização de eventos conjuntos. Em 2007, além dessas atividades, iniciou-se o atendimento odontológico destas famílias, na chamada "clínica do PSF", onde os projetos terapêuticos singulares eram discutidos por equipes de estudantes. Buscava-se a resolução do problema do paciente, levando-se em conta seu contexto familiar e social. Os estudantes participavam de reuniões com a equipe multiprofissional, da elaboração do genograma e do atendimento clínico odontológico.

Nesse cenário de políticas macro convergentes, de discussão ampliada e inclusiva, do apoio da gestão do Departamento e da presença de um grupo consciente da importância destas iniciativas, em 2009, a CA do Departamento de Odontologia da UEM foi iniciada, com uma proposta de modificar a lógica do atendimento e ter uma clínica-escola que ensinasse os estudantes a cuidar de pessoas, famílias e comunidade em uma abordagem integral. A CA é a porta de entrada para a atenção odontológica no curso de Odontologia da UEM e atende os usuários do SUS desde 2002, sendo um ponto de atenção à saúde bucal importante do município. A CA está inserida na disciplina de estágios supervisionados em saúde coletiva do último ano do curso de graduação (5o. ano), com a composição dos estudantes da graduação, pósgraduação, docentes, agentes universitários e usuários.

A CA da UEM tem como objetivo, além de oferecer um atendimento integral e humanizado ao paciente, o de promover a interação e o vínculo do estudante com os demais sujeitos envolvidos na atenção odontológica, como a comunidade e a equipe multiprofissional de saúde. $\mathrm{O}$ atendimento odontológico do paciente é realizado pelos estudantes, orientados por docentes de todas disciplinas. O planejamento é integrado e oferece opções ao usuário, que escolhe o tratamento conforme suas condições e expectativas. Assim, o usuário segue um fluxo de atendimento, de acordo com o grau de complexidade dos procedimentos previstos ${ }^{10,11}$. Além disso, o usuário apresenta um fluxo interno e externo para acompanhamento. Semanalmente, ocorrem reuniões com docentes, estudantes e agentes universitários, que integram a COPACA. Nessas reuniões, são trabalhados temas atinentes à avaliação, intervenção e reorientação das atividades e metas da clínica ${ }^{12}$. A formação dessa comissão teve como objetivo a busca da qualidade dos serviços de saúde bucal, produção de conhecimento e colegiadamente reorganizar o processo de trabalho no cotidiano da CA da UEM, extrapolando para as outras clínicas da 
graduação, outras necessidades de articulação e para o próprio ensino da Odontologia ${ }^{9}$.

Desta maneira, o objetivo deste trabalho foi relatar o processo de construção coletiva da CA, na Odontologia, por meio da gestão colegiada, durante o período de 2010 a 2017.

\section{METODOLOGIA}

Este estudo descritivo, de caráter documental $^{13}$, baseou-se na análise dos temas registrados nas atas das reuniões semanais da Comissão Permanente de Avaliação da CA (COPACA), do Departamento de Odontologia (DOD), da UEM, na cidade de Maringá/PR, desde o primeiro semestre de 2010 até o primeiro semestre de 2017.

Todos os documentos foram verificados por dois pesquisadores de forma independente (MF, NBR). Após análise, os temas registrados nas atas das reuniões foram agrupados.

Para a análise documental, recorreu-se a metodologia da análise de conteúdo ${ }^{14}$, que representa o conjunto de técnicas de investigação científica baseada em dados linguísticos. Nesse tipo de análise, os elementos fundamentais da comunicação são identificados, numerados e categorizados. As categorias devem, antes de tudo, refletir os propósitos da pesquisa. A análise documental das atas serviu de base para o conhecimento dos assuntos abordados nas reuniões, demonstrando a participação de todos os integrantes da COPACA, por meio de suas contribuições durante as reuniões.

Para relatar e avaliar o processo de construção coletiva da CA, cuja implantação nos serviços de saúde está incluída entre as propostas da $\mathrm{PNH}$, os dados levantados resultaram em 83 temáticas que, uma vez quantificadas e analisadas, foram agrupadas e classificadas em três famílias: CA, PNH e assuntos gerais.

Os dados selecionados são apresentados de forma descritiva.

\section{RESULTADOS}

Neste estudo, foram avaliadas todas as reuniões ocorridas no período de 2010 a 2017 (tabela 1), ou seja, desde a criação institucional da COPACA.

As análises dos documentos resultaram em 3 categorias que agregam a CA (tabela 2), política nacional de humanização (tabela 3) e assuntos gerais (tabela 4), distribuídas por ano.

Os assuntos classificados dentro da temática "CA" foram relacionados a implantação, organização, regulação, funcionamento, avaliação, corpo estruturante, planejamento, capacitação e divulgação da CA.

Os assuntos classificados dentro da temática "PNH" foram os relacionados com os princípios desta política, como: acolhimento, escuta do usuário, trabalho em equipe, gestão coparticipativa e planejamento clínico integrado, bem como discussões intersetoriais e visitas de consultores do Ministério da Saúde (MS) para acompanhamento das ações baseadas na PNH.

Os assuntos classificados na temática "assuntos gerais" são os relacionados ao Departamento e à Clínica de Odontologia, que envolvem discussões a respeito do perfil do egresso que temos interesse de formar. Além disso, são discutidos problemas de outros setores, experiências de docentes e estudantes em congressos e reuniões externas, planejamento financeiro e resolução de problemas técnicos da clínica. Vale salientar que a CA também funciona como espaço para análise e reflexão sobre assuntos atinentes à Odontologia, de uma maneira geral, como, por exemplo, a contribuição do cirurgião-dentista na notificação compulsória de doenças, bem como de casos de violência doméstica.

Como produtos das discussões e construções coletivas realizadas pela COPACA, podemos citar 3 documentos básicos: 1) Prontuário único; 2) Manual da Clínica 
Ampliada; 3) Instrutivo da Clínica Ampliada.

O Prontuário Único do Paciente foi elaborado com a participação de docentes de todas as especialidades. O Manual da Clínica Ampliada, produzido em 2010, contém o fluxograma da clínica, a descrição da operacionalização do atendimento, as fases do planejamento integrado que auxiliam o estudante na construção do plano de tratamento, os critérios para a indicação dos procedimentos de acordo com as séries, áreas e grau de complexidade, as fichas de referência e contrarreferência e a ficha de alta.

O Instrutivo da Clínica Ampliada contém a nova ficha de avaliação do desempenho dos estudantes (contemplando os propósitos de avaliação por competência), o painel com as informações sobre a organização do atendimento na Clínica Odontológica da UEM, o questionário de satisfação do usuário pré e pós-atendimento, as normas para os seminários de planejamento integrado de casos clínicos, a composição das equipes de trabalho e o Regulamento da Clínica Ampliada, bem como informações sobre o papel do controle social no atendimento odontológico.

A Clínica Ampliada da UEM foi visitada por membros da PNH do Ministério da Saúde desde a sua criação. Os registros das falas das consultoras e apoiadoras da PNH nas reuniões da COPACA mostraram que a CA do Curso de Odontologia da UEM tem sido uma experiência pioneira e inovadora na área da Odontologia e que a divulgação dos seus avanços traria benefícios potenciais à comunidade acadêmica e aos serviços de saúde.

A COPACA também discutiu e deliberou sobre assuntos atinentes à operacionalização da Clínica Odontológica.

Tabela 1 - Frequência absoluta e relativa do número de reuniões da COPACA realizadas em cada ano.

\begin{tabular}{ccc}
\hline ANO & Frequência Absoluta & Frequência Relativa \\
\hline $\mathbf{2 0 1 0}$ & 33 & $18.33 \%$ \\
$\mathbf{2 0 1 1}$ & 26 & $14,45 \%$ \\
$\mathbf{2 0 1 2}$ & 23 & $12,78 \%$ \\
$\mathbf{2 0 1 3}$ & 22 & $12,22 \%$ \\
$\mathbf{2 0 1 4}$ & 23 & $12,78 \%$ \\
$\mathbf{2 0 1 5}$ & 15 & $8,33 \%$ \\
$\mathbf{2 0 1 6}$ & 23 & $12,78 \%$ \\
$\mathbf{2 0 1 7}$ & 15 & $8,33 \%$ \\
\hline TOTAL & $\mathbf{1 8 0}$ & $\mathbf{1 0 0 \%}$ \\
\hline
\end{tabular}


Tabela 2 - Resultado da análise dos dados sobre a temática "Clínica Ampliada”, com seus respectivos assuntos nos anos de 2010 a 2017.

\begin{tabular}{|c|c|c|c|c|c|c|c|c|}
\hline Assuntos CA & 2010 & 2011 & 2012 & 2013 & 2014 & 2015 & 2016 & 2017 \\
\hline Capacitação sobre o funcionamento da CA (fluxos e atendimentos) & $\mathrm{X}$ & $\mathrm{X}$ & $\mathrm{X}$ & & & & $\mathrm{X}$ & $\mathrm{X}$ \\
\hline Projeto terapêutico singular & $\mathrm{X}$ & & & $\mathrm{X}$ & $\mathrm{X}$ & & & $\mathrm{X}$ \\
\hline Regulamento da CA & $\mathrm{X}$ & $\mathrm{X}$ & $\mathrm{X}$ & & & & & \\
\hline Pactuação das regras na CA com os alunos da graduação & & & $\mathrm{x}$ & $\mathrm{X}$ & $\mathrm{X}$ & & & \\
\hline Desenvolvimentos de documentos legais para atendimento do paciente & & $\mathrm{X}$ & & & & & & $\mathrm{X}$ \\
\hline Discussão sobre a avaliação dos alunos & $\mathrm{X}$ & & $\mathrm{X}$ & & $\mathrm{X}$ & $\mathrm{X}$ & $\mathrm{X}$ & $\mathrm{X}$ \\
\hline Discussão sobre a biossegurança & & & & & $\mathrm{X}$ & $\mathrm{X}$ & $\mathrm{X}$ & $\mathrm{X}$ \\
\hline Discussão sobre a importância dos determinantes sociais no processo saúde-doença & & $\mathrm{X}$ & & & & & & \\
\hline Discussão sobre as ausências de pacientes & $\mathrm{X}$ & $\mathrm{X}$ & $\mathrm{X}$ & & $\mathrm{X}$ & & & \\
\hline Discussão sobre o corpo clínico da CA e COPACA & $\mathrm{X}$ & & $\mathrm{X}$ & & $\mathrm{X}$ & & & \\
\hline Discussão sobre o papel da CA & & $\mathrm{X}$ & & $\mathrm{X}$ & $\mathrm{X}$ & & $\mathrm{X}$ & \\
\hline Envolvimento da pós-graduação na CA & $\mathrm{X}$ & $\mathrm{X}$ & $\mathrm{X}$ & $\mathrm{X}$ & $\mathrm{X}$ & $\mathrm{X}$ & $\mathrm{X}$ & $\mathrm{X}$ \\
\hline Escuta da opinião dos alunos de graduação e pós-graduação & $\mathrm{X}$ & $\mathrm{X}$ & $\mathrm{X}$ & $\mathrm{X}$ & $\mathrm{X}$ & $\mathrm{X}$ & $\mathrm{X}$ & $\mathrm{X}$ \\
\hline Fluxograma de atendimento & $\mathrm{X}$ & & & & $\mathrm{X}$ & & $\mathrm{X}$ & $\mathrm{X}$ \\
\hline Instrutivo da CA & & $\mathrm{X}$ & & & & & $\mathrm{X}$ & \\
\hline Modificações nos prontuários e seu preenchimento & $\mathrm{X}$ & & & & $\mathrm{X}$ & $\mathrm{x}$ & & \\
\hline Planejamento para o próximo ano da CA & $\mathrm{X}$ & $\mathrm{x}$ & $\mathrm{x}$ & $\mathrm{X}$ & $\mathrm{X}$ & $\mathrm{X}$ & $\mathrm{X}$ & \\
\hline Procedimento clínico mínimo realizado CA & $\mathrm{X}$ & & & & & $\mathrm{X}$ & $\mathrm{X}$ & $\mathrm{X}$ \\
\hline Discussão das normas dos Seminários da CA & & $\mathrm{X}$ & $\mathrm{X}$ & $\mathrm{X}$ & $\mathrm{X}$ & $\mathrm{X}$ & $\mathrm{X}$ & $\mathrm{X}$ \\
\hline Realização de workshops com metodologias ativas de ensino aprendizagem & $\mathrm{X}$ & $\mathrm{X}$ & $\mathrm{X}$ & $\mathrm{X}$ & $\mathrm{X}$ & $\mathrm{X}$ & $\mathrm{X}$ & $\mathrm{X}$ \\
\hline Utilização de redes sociais & $\mathrm{X}$ & & & & & & & \\
\hline
\end{tabular}


Tabela 3 - Resultado da análise dos dados sobre a temática "PNH”, com seus respectivos assuntos nos anos de 2010 a 2017.

\begin{tabular}{|c|c|c|c|c|c|c|c|c|}
\hline Assuntos PNH & 2010 & 2011 & 2012 & 2013 & 2014 & 2015 & 2016 & 2017 \\
\hline Aplicação dos princípios da PNH na prática clínica & $\mathrm{X}$ & & & & $\mathrm{X}$ & & & $\mathrm{x}$ \\
\hline Desenvolvimento e discussão Protocolo de ACCR urgência & & $\mathrm{x}$ & & $\mathrm{X}$ & & $\mathrm{x}$ & & \\
\hline Discussão Protocolo de ACCR da CA & & & & & & $\mathrm{x}$ & $\mathrm{X}$ & $\mathrm{X}$ \\
\hline Discussões de ações intersetoriais para aplicação da PNH & $\mathrm{X}$ & & & & $\mathrm{X}$ & & & \\
\hline Escuta do usuário do SUS & & $\mathrm{x}$ & & $\mathrm{X}$ & & & & \\
\hline Estímulo ao trabalho em equipe & $\mathrm{x}$ & $\mathrm{x}$ & $\mathrm{X}$ & $\mathrm{X}$ & $\mathrm{X}$ & $\mathrm{x}$ & $\mathrm{x}$ & $\mathrm{x}$ \\
\hline Gestão coparticipativa & $\mathrm{x}$ & $\mathrm{x}$ & $\mathrm{x}$ & $\mathrm{X}$ & $\mathrm{x}$ & $\mathrm{x}$ & $\mathrm{X}$ & $\mathrm{x}$ \\
\hline Planejamento clínico integrado & $\mathrm{x}$ & $\mathrm{x}$ & $\mathrm{x}$ & $\mathrm{X}$ & $\mathrm{x}$ & $\mathrm{X}$ & $\mathrm{X}$ & \\
\hline Reunião com consultora do Ministério da saúde sobre PNH & $\mathrm{x}$ & $\mathrm{X}$ & $\mathrm{X}$ & & $\mathrm{X}$ & $\mathrm{x}$ & & \\
\hline
\end{tabular}

Tabela 4 - Resultado da análise dos dados sobre a temática “assuntos gerais”, com seus respectivos assuntos nos anos de 2010 a 2017.

\begin{tabular}{|c|c|c|c|c|c|c|c|c|}
\hline Assuntos gerais & 2010 & 2011 & 2012 & 2013 & 2014 & 2015 & 2016 & 2017 \\
\hline Formação do perfil do aluno de acordo com DCN & $\mathrm{X}$ & $\mathrm{x}$ & & $\mathrm{x}$ & & $\mathrm{x}$ & $\mathrm{X}$ & $\mathrm{x}$ \\
\hline Discussão e decisões sobre outros setores de interesse do DOD & $\mathrm{x}$ & $\mathrm{x}$ & & $\mathrm{X}$ & $\mathrm{X}$ & $\mathrm{x}$ & $\mathrm{X}$ & $\mathrm{X}$ \\
\hline Discussão sobre violência e notificação & & $\mathrm{x}$ & & $\mathrm{x}$ & & & & \\
\hline Experiências compartilhadas de participação em congresso e cursos & $\mathrm{X}$ & $\mathrm{X}$ & $\mathrm{X}$ & $\mathrm{X}$ & & & $\mathrm{X}$ & $\mathrm{X}$ \\
\hline Planejamento financeiro & $\mathrm{X}$ & & & & & & & $\mathrm{x}$ \\
\hline Resolução de problemas técnicos da clínica & & & & & $\mathrm{X}$ & & $\mathrm{x}$ & $\mathrm{X}$ \\
\hline
\end{tabular}




\section{DISCUSSÃO}

Inicialmente, a COPACA teve como objetivo sensibilizar e informar docentes e funcionários sobre os novos processos de trabalho. Em um segundo momento, serviu de apoio para a implementação e a organização da clínica ampliada, bem como para aumentar o comprometimento dos atores envolvidos. Mais recentemente, nas reuniões dessa comissão, as mudanças são validadas e os rumos são, continuamente, repensados.

A implantação da CA significou um olhar verdadeiramente "ampliado" para o usuário, que deixou de ser mero instrumento de treinamento técnico. Sensibilidade e ética para a escuta qualificada, bem como abertura para o diálogo e para a identificação das necessidades individuais têm sido requisitos de um atendimento humanizado. Antes da implantação da CA e da elaboração de um prontuário único para o usuário, o paciente possuía fichas clínicas separadas para as diferentes especialidades da Odontologia e, muitas vezes, era atendido de acordo com a necessidade de aprendizagem do aluno.

Para que as mudanças ocorressem, foram necessários a participação e o empenho de todo o corpo docente, já que a implantação da CA também modificou a organização das demais clínicas da graduação, não só pela adoção de um prontuário único, mas também porque o fluxo de atendimento passou a se estabelecer a partir da acolhida na CA. A autonomia dos usuários na manutenção de sua saúde bucal e geral passou a ser o foco do cuidado, e não mais apenas as demandas de atendimento clínico.

Desde a criação da COPACA, as reuniões têm sido semanais. Na tabela 1, percebe-se uma redução do número de reuniões a partir do ano de 2011, porque foram inseridos os seminários de planejamento integrado de casos clínicos. Os seminários são espaços para a exposição e discussão dos casos clínicos integrados em auditórios específicos para acomodar os alunos de graduação, docentes, agentes universitários e preceptores. Assim, nos dias de seminário, não há tempo para a reunião da COPACA. Já em 2015, houve uma queda em função de uma paralisação das atividades na UEM. Em 2017, o período analisado foi o primeiro semestre. A periodicidade semanal tem oportunizado reflexão e avaliação permanentes, bem como resolução de intercorrências.

Os processos de qualificação do pessoal da saúde devem ser estruturados a partir da problematização do seu processo de trabalho, com o objetivo de transformação das práticas profissionais e da própria organização do trabalho, tomando como referência as necessidades de saúde das pessoas e das populações, da gestão setorial e do controle social em saúde. Desse modo, transformar a formação e a gestão do trabalho em saúde não podem ser consideradas questões simplesmente técnicas, já que envolvem mudança nas relações, nos processos, nos atos de saúde e, principalmente, nas pessoas. São questões tecnopolíticas e implicam a junção de ações para dentro e para fora das instituições de saúde, na perspectiva de ampliação da qualidade da gestão, do aperfeiçoamento da integralidade, da competência do conceito ampliado de saúde e do fortalecimento do controle da sociedade no sistema9 ${ }^{9}$. Estes são os objetivos da gestão colegiada da Clínica Ampliada da Odontologia da UEM. A concretização da Clínica Ampliada na UEM, com o necessário envolvimento de docentes, agentes universitários e acadêmicos da graduação e pós-graduação teve o intuito de qualificar o serviço e, consequentemente, a formação dos futuros profissionais. Essa experiência tem oferecido, aos alunos de graduação e pós-graduação do curso de Odontologia da UEM, subsídios para uma nova 
visão do cuidado integral ao usuário.

A $\mathrm{PNH}^{3}$ tem como princípios a transversalidade, a indissociabilidade entre gestão e atenção, bem como o protagonismo, a corresponsabilidade e a autonomia dos sujeitos envolvidos. Como diretrizes da PNH, destacamos o acolhimento, a gestão participativa, a ambiência, a Clínica Ampliada, a valorização do trabalhador e a defesa dos direitos dos usuários $^{3}$. Essa política tem como propostas: 1) a obrigação com o sujeito e não com a doença; 2) o reconhecimento dos limites dos saberes, com a afirmação de que o sujeito é sempre maior do que o diagnóstico proposto; 3) a afirmação do encontro clínico entre o trabalhador de saúde e o usuário; 4) a busca pelo equilíbrio entre os danos e os benefícios gerados pelas práticas de saúde; 5) a aposta nas equipes multiprofissionais $\mathrm{e}$ transdisciplinares; 6) a corresponsabilidade entre diferentes sujeitos; e 7) a defesa dos direitos dos usuários $^{5,15}$. Neste contexto, a Clínica Ampliada da UEM tem proporcionado a vivência de uma gestão colegiada e participativa, que se traduz pela valorização de cada ator e pelo desenvolvimento do potencial individual para sua construção coletiva. Gestão participativa ou cogestão é o modo de administrar que não se limita pela linha superior de comando e que inclui o pensar e o fazer coletivos. É centrada no trabalho em equipe, na construção coletiva (planeja quem executa) e em espaços coletivos que garantam o compartilhamento do poder ${ }^{16}$. A COPACA do DOD da UEM se assemelha ao que é citado no estudo de Rios (2009)², já que configura um espaço transdisciplinar para discussão e resolução das situações de impasse, sofrimento, angústia e desgaste a que se submetem os profissionais de saúde na sua rotina de trabalho.

Um assunto que causou bastante preocupação nas reuniões da COPACA foi o acolhimento: como acolher de forma humanizada, identificar prioridades, manter um fluxograma de atendimento e diminuir as filas de espera $^{17}$. Nessas discussões, foi definido o "Acolhimento com Classificação de Risco" para o setor de urgência da Clínica Odontológica da UEM e, em 2016, foi iniciada a discussão sobre a possibilidade de adoção na clínica ampliada também.

O acolhimento é a escuta do usuário em suas queixas, no reconhecimento do seu protagonismo no processo de saúde e adoecimento, e na responsabilização pela resolução, com ativação de redes de compartilhamento de saberes. Acolher é um compromisso de resposta às necessidades dos cidadãos que procuram os serviços de saúde. Não tem hora para acontecer. Ele está presente em todas as fases do atendimento do paciente nos serviços de saúde 5 . O objetivo do acolhimento com estratificação de risco foi permitir a priorização do atendimento aos que têm maior risco e atividade de doença. $\mathrm{O}$ profissional deve levar em consideração os fatores determinantes de risco, não só biológicos e odontológicos, mas também os comportamentais, culturais e socioeconômicos ${ }^{18}$.

A proatividade do usuário é uma ferramenta essencial para que este seja atendido de forma integral. Por meio dela, é possível a construção de vínculos, o respeito à diversidade e à singularidade no encontro entre quem cuida $\mathrm{e}$ quem recebe o cuidado ${ }^{19}$.

Na Clínica Ampliada, os trabalhadores de saúde buscam trabalhar estratégias para que o paciente ganhe autonomia, conversando sobre as possíveis causas do problema, o contexto de vida do paciente, ressaltando a importância do autocuidado em saúde bucal para estimular que os usuários consigam manter sua saúde bucal quando não estiverem em tratamento odontológico.

Para a resolutividade dos casos, os 
profissionais na CA constroem a corresponsabilidade do cuidado, trocando saberes, emoções, sentimentos, crenças, enfim, muito mais que apenas os dados dos sinais, sintomas e resultados de exames ${ }^{20}$. Escutar o paciente é dar sempre oportunidades ao mesmo de dizer o que pensa, no momento clínico ou também por meio de ouvidoria, caixas de opinião e pesquisas de satisfação ${ }^{21}$. A COPACA desenvolveu um questionário de satisfação que o usuário preenche quando recebe alta do serviço.

$\mathrm{Na} \mathrm{CA}$, em todos os anos consultados, houve um constante exercício do relacionamento interpessoal. $\mathrm{O}$ saber ouvir e construir coletivamente, de forma lenta e progressiva, como base para as mudanças crescentes e constantes, tem sido pautado nas normativas do Ministério da Saúde e do Ministério da Educação, apoiados pela $\mathrm{PNH}^{5}$. A Política Nacional de Humanização deve se fazer presente e estar inserida em todas as políticas e programas do SUS. Ela busca transformar as relações de trabalho a partir do aumento do contato e da comunicação entre pessoas e grupos, tirando-os do isolamento e das relações de poder hierarquizadas. A transversalização é o reconhecimento de que as diferentes especialidades e ações na saúde podem conversar com a experiência daquele que é assistido e, em conjunto, os conhecimentos podem produzir saúde de forma mais corresponsável ${ }^{15}$.

A análise das atas deixa claro que houve, desde o princípio, preocupação em envolver a pós-graduação nas atividades da CA, tanto na orientação e no acompanhamento dos estudantes em clínica, como no desenvolvimento dos seminários para discussão dos casos. Isso viabilizou uma integração efetiva entre graduação e pós-graduação.

O prontuário único foi criado em 2010 e consiste no conjunto de documentos relativos à assistência prestada a um paciente. Ele contém todas as informações sobre a saúde do paciente, as anotações dos profissionais que realizaram 0 atendimento, os resultados dos exames e os relatórios dos procedimentos e dos tratamentos. Há valor legal e ético perante a Justiça, pois ele reúne informações sigilosas de caráter médico, científico e legal (jurídico) ${ }^{22}$. O prontuário clínico único da $\mathrm{CA}$ foi construído coletivamente e busca uma abordagem integrada do caso clínico, visando à integralidade da atenção, pensando na referência e contrarreferência com o município, e também vislumbrando o encaminhamento para outros níveis de atenção, como o Centro de Especialidades Odontológicas ${ }^{10}$. Apresenta vantagens pois enxerga o paciente como um todo, e não de forma fragmentada por especialidade, além de centralizar as informações.

Em todos os passos para as inúmeras mudanças conseguidas para a construção da CA do Curso de Odontologia da UEM, podemos destacar os esforços da área de Saúde Coletiva, que possibilitaram a aproximação das demais áreas da Odontologia.

Uma tradução possível para o significado do trabalho na CA na UEM, realizado pela COPACA, está contida no verbo tecer. Tecer, abrir, começar, costurar, pintar, unir, fiar e entrelaçar. É o reflexo de um novo modo de trabalhar, uma forma diferente de tecer as sutis relações humanas, profissionais, de saberes, do ensino com o serviço e a comunidade, visando uma atenção integral. Um desafio e tanto que tem sido o norte daqueles que lutam pela consolidação do SUS.

Algumas limitações do estudo devem ser ressaltadas. Primeiramente, trata-se de uma análise documental, que não teve o objetivo de responder a questões de pesquisa, mas de fazer o registro das temáticas das discussões. Outra limitação se deve ao fato da análise ter sido realizada por docentes que faziam parte do processo de construção da CA.

As percepções individuais dos membros da COPACA sobre o processo de construção coletiva 
por meio da gestão colegiada poderiam ser coletadas a partir de uma abordagem qualitativa.

\section{CONCLUSÃO}

Este estudo permitiu concluir que a $\mathrm{CA}$, ao longo desses sete anos, tem estado em contínua construção e aprimoramento, e isso graças a um esforço coletivo de todos os envolvidos. As reuniões semanais da COPACA são o espaço onde a gestão colegiada se consolida e a coletividade da construção é legitimada. Diversas temáticas emergiram a partir de problemas detectados no dia a dia. Houve discussão e reflexão, e propostas foram elaboradas por esse colegiado (COPACA), sempre objetivando a melhoria do serviço e do ensino, conforme preconiza a PNH.

\section{ABSTRACT \\ Process of collective construction of the Expanded Clinic in Dentistry}

The objective of this study was to report the collective construction process of the Expanded Clinic (EC) in Dentistry, through permanent health education. This descriptive study is a documentary based in the analysis of the topics in the records of the weekly meetings of the Permanent Commission for the Evaluation of the Expanded Clinic (CEEC), Department of Dentistry, State University of Maringá (UEM), from the first half of 2010 to the first half of 2017. The data were interpreted using the content analysis method recommended by Bardin. The documents pointed out that the impacts of collegial management on the UEM dental clinic's operation were: greater problem solving, less difficulty in confrontations and greater commitment, more efficient case planning and satisfaction of all in this collective construction process. As products of the collective discussions and constructions carried out by CEEC, we can cite three basic documents: Single record; Expanded Clinic's Instructional and Manual. This study allowed us to conclude that the construction and improvement of the EC have occurred continuously over seven years, resulting from an effort and synergy of the team. The weekly meetings of CEEC are the space where collegial management is consolidated and listening, and potentialities are legitimized. Several issues emerged from problems detected daily. There were discussion and reflection, and proposals were elaborated aiming at the improvement of service and teaching, as recommended by the National Humanization Policy.

Descriptors: Humanization of Assistance. Dentistry. Education, Continuing. Brazilian Health System.

\section{REFERÊNCIAS}

1. Brasil. Ministério da Saúde. HumanizaSUS: Documento base para gestores e trabalhadores do SUS. 4. ed. Brasília: Editora do Ministério da Saúde, 2010.

2. Rios IC. Humanização: a essência da ação técnica e ética nas práticas de saúde. Rev Bras Educ Med. 2009; 33(2):253-61.

3. Ministério da Saúde (Brasil). Política nacional de humanização: a humanização como eixo norteador das práticas de atenção e gestão em todas as instâncias do SUS. Brasília: Editora do Ministério da Saúde, 2004.

4. Brasil. Ministério da Saúde. Clínica Ampliada, equipe de referência e projeto terapêutico singular. $2^{\mathrm{a}}$ ed. Brasília: Editora do Ministério da Saúde; 2007.

5. Brasil. Ministério da Saúde. Acolhimento nas práticas de produção de saúde. 2. ed. 5. reimp. Brasília: Editora do Ministério da Saúde, 2010.

6. Terada RSS, Nakama L (Org). A implantação das diretrizes curriculares nacionais de Odontologia: a experiência de Maringá. São Paulo/Londrina/Brasília :HUCITEC/Rede Unida/Abeno, 180p. 2004.

7. Hayacibara MF, Terada RSS, Silva MC, Morita MC, Tanaka E, Krieger L. II Fórum dos projetos Pró-Saúde Odontologia das universidades do estado do Paraná - relato de experiência. Rev Espaço para a Saúde. 2009; 
11(1): 54-60.

8. Brasil. Ministério da Saúde. A Educação Permanente Entra na Roda: pólos de educação permanente em saúde - conceitos e caminhos a percorrer. Brasília: Ministério da Saúde, 2005.

9. Cecim RB, Feuerwerker LCM. O Quadrilátero da Formação para a Área da Saúde: Ensino, Gestão, Atenção e Controle Social. Rev Saúde Colet. 2004, 14(1):41- 65.

10. Hayacibara MF, Lolli LF, Terada RSS, Hidalgo MM, Bispo CGC, Terada HH, et al. Experiência de Clínica Ampliada em Odontologia na Universidade Estadual de Maringá. Rev Bras Educ Méd. 2012; 36(1):178-83.

11. Fujimaki M. Clínica Ampliada - A Odonto Além da Boca, uma experiência em Maringá PR. Documentário. [Acesso em 5 out. 2017]. Disponível em: http://redehumanizasus.net/ clinica-ampliada-a-odonto-alem-da-bocauma-experiencia-em-maringa-pr/.

12. Peruchi CTR, Souza AP, Hidalgo MM, Terada RSS, Peixoto IS, Bispo CGC. A Clínica Ampliada na Odontologia: avaliação do trabalho clínico onde o ensino acontece. Rev Assoc Paul Cir Dent. 2015;69(2):170-7.

13. Marcone MA, Lakatos EM. Técnicas de Pesquisa: planejamento e execução de pesquisas, amostragens e técnicas de pesquisa, elaboração, análise e interpretação de dados. 3 ed. São Paulo: Atlas; 1999.

14. Bardin L. Análise de conteúdo. São Paulo: Edições 70; 2011.

15. Brasil. Folheto da Política Nacional de Humanização - PNH. 1. ed. 1 reimp. Brasília: Editora do Ministério da Saúde, 2013.

16. Brasil. Ministério da Saúde. Gestão participativa e cogestão / Ministério da Saúde, Secretaria de Atenção à Saúde, Política Nacional de Humanização da
Atenção e Gestão do SUS. Brasília: Editora do Ministério da Saúde, 2009.

17. Padilha LS. Processo de implantação do acolhimento com classificação de risco no setor de urgências da clínica de odontologia da UEM Dissertação de Mestrado. Departamento de Odontologia. Universidade Estadual de Maringá. Maringá, 2012. [Acesso em 5 out. 2017]. Disponível em: http://nourau.uem.br/nourau/document $/$ ?code $=$ vtls 00 $\underline{0213963 .}$.

18. Paraná. Secretaria de Estado da Saúde do Paraná. Superintendência de Assistência à Saúde. Linha guia de saúde bucal. - Curitiba: SESA, 2014.

19. Maynart WH, Albuquerque MC, Brêda MZ, Jorge JS. A escuta qualificada e o acolhimento na atenção psicossocial. Acta Paul Enferm. 2014; 27(4):300-3.

20. Zoboli ELCP. Bioética e atenção básica: para uma clínica ampliada, uma Bioética clínica amplificada. O Mundo da Saúde São Paulo: 2009; 33(2):195-204.

21. Bispo CGC, Hidalgo MM, Correa VL, Calazans CM, Iwaki Filho L, Hayacibara $\mathrm{MF}$ et al. Estratégias para humanização propostas pelos alunos do quarto ano do Curso de Odontologia da Universidade Estadual de Maringá. Rev Med Minas Gerais 2009, 19 (4Suppl 2), S59-63.

22. Conselho Regional de Medicina. RESOLUÇÃO CFM no 1.638/2002. Define prontuário médico e torna obrigatória a criação da Comissão de Revisão de Prontuários nas instituições de saúde. Publicada no D.O.U. 2002, Seção I, p.184-5.

Correspondência para

Najara Barbosa Rocha

e-mail: najara.rocha@gmail.com

Av. Mandacaru, 1550. Bloco S08

87083-170 Maringá/PR 